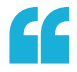

in several

disease

models it may

be caspase 11 ,

rather than

caspase 1 ,

that drives

pathological

inflammation

\title{
A new path uncovers a wrongful conviction
}

Inflammasomes serve as scaffolds to promote the activation of caspase 1 and the release of interleukin- $1 \beta$ (IL-1 $\beta$ ) and IL-18 in response to various pro-inflammatory stimuli. Now, Vishva Dixit and co-workers have described a 'non-canonical' inflammasome, which engages caspase 11 to trigger the caspase 1-dependent production of pro-inflammatory cytokines and caspase 1-independent pyroptosis.

The authors were exploring how cholera enterotoxin subunit B (CTB) promotes inflammasome activation in bone marrow-derived macrophages primed with lipopolysaccharide (LPS). They showed that this process results in the processing of caspase 1 in a manner dependent on NOD-, LRR- and pyrin domain-containing 3 (NLRP3) and the adaptor protein ASC. However,

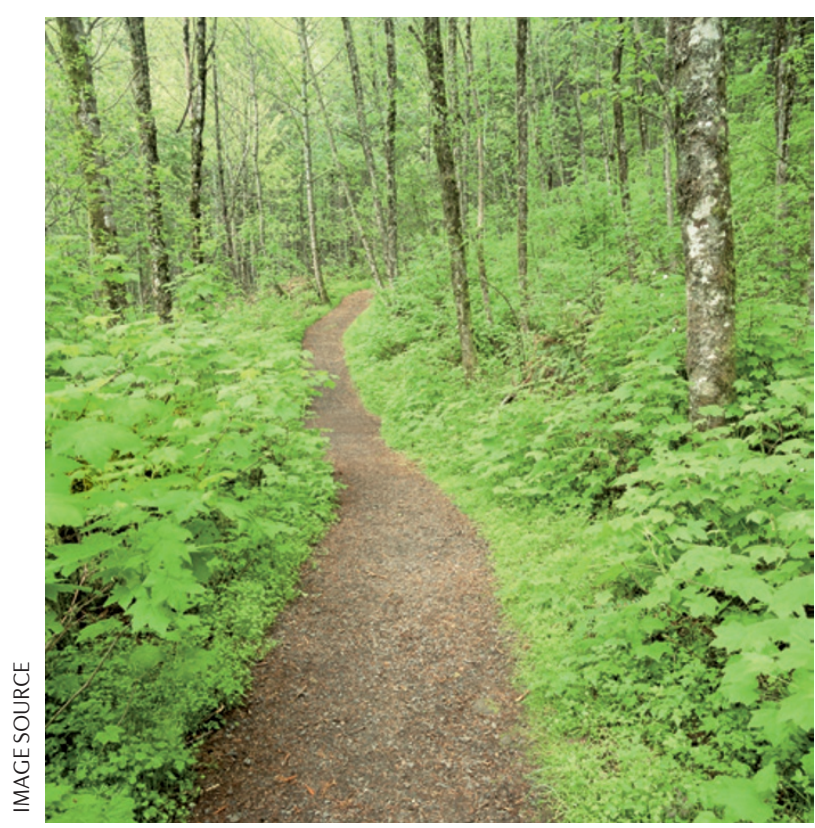

comparing inflammasome activation in different mouse strains, they were surprised to find that macrophages from mice of strain 129 did not secrete IL- $1 \beta$ in response to CTB. Screening experiments indicated that strain 129 mice have a deficient copy of the Casp11 gene, and LPS-primed macrophages from caspase 11deficient C57BL/6 mice also failed to secrete IL- $1 \beta$ in response to CTB, despite responding to canonical activators of the inflammasome, such as ATP, double-stranded DNA and flagellin. Caspase 11-deficient C57BL/6 macrophages secreted IL- $1 \beta$ following infection with Francisella tularensis or Pseudomonas aeruginosa, but were unable to produce IL-1 $\beta$ after infection with Escherichia coli, Citrobacter rodentium or Vibrio cholerae. This led the authors to propose the existence of a non-canonical inflammasome, which activates caspase 1 downstream of caspase 11 engagement.

An important outcome of inflammasome assembly and caspase 1 activation is the induction of pyroptosis, an inflammatory type of cell death. The authors found that caspase 11 was not required for macrophage pyroptosis in response to canonical activators of the inflammasome, but was necessary for the induction of pyroptosis following infection with E. coli, C. rodentium or V. cholerae. Interestingly, NLRP3 and ASC were not needed for caspase 11 to deliver death signals in response to these bacteria. Addressing the role of caspase 1 in non-canonical inflammasome activation, the authors made a rather startling finding. All caspase 1-deficient mice were initially generated on a 129 background and, owing to the proximity of Casp1 and Casp11 in the mouse genome, have retained the defective 129-derived Casp11, despite many generations of backcrossing. To circumvent this difficulty, bacterial artificial chromosomes were used to restore functional Casp11 expression in caspase 1-deficient mice. Using these mice, the authors showed that during non-canonical inflammasome activation caspase 1 is required for IL- $1 \beta$ processing but not for macrophage pyroptosis.

Finally, the authors assessed the relative contributions of caspase 1 and caspase 11 in a model of LPS-induced septic shock. Both caspase 11-deficient mice and the double-deficient mice showed increased survival compared with wild-type controls, but mice that were only deficient for caspase 1 were not protected. By contrast, previous studies that had inadvertently used the double-deficient mice suggested that caspase 1 contributed to LPS-induced lethality. Thus, the authors' findings are notable, not only for their description of this non-canonical pathway of inflammasome activation, but also because they highlight the need to re-address other findings made in the original caspase 1-deficient mice. Dixit and colleagues propose that in several disease models it may be caspase 11, rather than caspase 1 , that drives pathological inflammation.

Yvonne Bordon

ORIGINAL RESEARCH PAPER Kayagaki, N. et al. Non-canonical inflammasome activation targets caspase-11. Nature 479, 117-121 (2011) 